\title{
Application of Straw Tube Detectors to Hyperon Studies
}

\section{S. JOWZAEE ${ }^{a, b, *}$ for the COSY-TOF collaboration}

${ }^{a}$ Institute of Physics, Jagiellonian University, St. Łojasiewicza 11, 30-348 Kraków, Poland

${ }^{b}$ Jülich Center for Hadron Physics, Forschungszentrum Jülich, 52428 Jülich, Germany

A high statistics measurement of the $\vec{p} \mathrm{p} \rightarrow \mathrm{pK}^{+} \Lambda$ reaction was done with the COSY-TOF experiment. The proton beam was polarized up to more than $80 \%$, and its momentum was $2.95 \mathrm{GeV} / c$. The reconstruction of the $\mathrm{pK}^{+} \Lambda$ events was done only with the straw tube tracker due to the higher spatial resolution of the straw tube tracker compared to other subdetectors. The Dalitz plot of the $\mathrm{pK}^{+} \Lambda$ events was analyzed to understand the reaction mechanism and presented in this paper. The Dalitz plot shows that the COSY-TOF experiment provides complete kinematic coverage of the phase space of this reaction which made it ideal for $\vec{p} p \rightarrow p K^{+} \Lambda$ reaction analysis. The influence of different physical effects as $\mathrm{p} \Lambda$ final state interaction, excited nucleon states ( $\mathrm{N}^{*}$-resonances) and a coupled channel effect of $\mathrm{N} \Sigma-\mathrm{p} \Lambda$ (N $\Sigma$ cusp effect) produce significant structures in the Dalitz plot. The angular distribution of the particles in the CMS and helicity frame was also presented in this paper.

DOI: 10.12693/APhysPolA.127.1531

PACS: 14.20.Jn, 13.75.Cs, 29.40.Gx

\section{Introduction}

Hyperons studies are interesting since there are strange quarks in the structure of these particles. The strange quark does not exist as constituent in the nucleon structure. In order to understand their properties, hyperons are produced in high energy physics experiments. Nucleon-nucleon reaction of $\overrightarrow{\mathrm{p} p} \rightarrow \mathrm{pK}^{+} \Lambda$ is one of the fundamental reaction to study the $\Lambda$-hyperon. Since there is no strangeness at the initial state of the reaction, it is possible to understand how the strangeness is created and how it decays.

Different $\vec{p} \mathrm{p} \rightarrow \mathrm{pK}^{+} \Lambda$ measurements have been done with polarized and unpolarized proton beams at different momenta from 2.7 to $3.3 \mathrm{GeV} / c$ in the COSY-TOF experiment [14]. The COSY-TOF is an ideal detector to measure the $\overrightarrow{\mathrm{p} p} \rightarrow \mathrm{pK}^{+} \Lambda$ reaction. The large acceptance of the detector in addition to the outstanding tracking capability and high resolution of the straw tube tracker allows a complete measurement of the $\mathrm{pK}^{+} \Lambda$ final states. The COSY-TOF detector kinematically covers the full phase space of the $\vec{p} p \rightarrow \mathrm{pK}^{+} \Lambda$ reaction and hence, the analysis of the Dalitz plot over the full phase space gets possible. Therefore, different effects involved in this reaction mechanism such as excited nucleon resonances $\left(\mathrm{N}^{*}\right), \mathrm{N} \Sigma-\mathrm{p} \Lambda$ coupled channel effect called $\mathrm{N} \Sigma$ cusp and the hyperon-nucleon final state interaction (FSI) close to the threshold of the reaction can be analyzed.

\section{Experimental setup}

The COSY-TOF detector is one of the external experiment at the Jülich COoler SYnchrotron COSY. The straw tube tracker (STT) is the main tracker in the COSY-TOF detector which has been installed about $30 \mathrm{~cm}$ behind the target. It consists of 2704 straw tubes organized in 13 double layers with three orientations with $60^{\circ}$ to each other for 3D track reconstruction. The new measurement with the COSY-TOF detector presented here are done with high statistics and a polarized proton beam at $2.95 \mathrm{GeV} / c$ momentum to study the $\vec{p} \mathrm{p} \rightarrow \mathrm{pK}^{+} \Lambda$ reaction mechanism. The reconstruction of the $\mathrm{pK}^{+} \Lambda$ final states was done only with the straw tube tracker, since the momentum resolution of the reconstructed primary

\footnotetext{
*e-mail: ajowzaee@gmail.com
}

particles with the STT alone is better than that from the time of flight analysis [3]. The $\mathrm{pK}^{+} \Lambda$ event can be uniquely determined by the event geometry in the absence of time of flight information. Using the direction of the measured particles, the $\Lambda$ decay vertex and the masses of all particles a kinematic fit is performed on the events to reduce the background which is mainly due to the $\overrightarrow{\mathrm{p} p} \rightarrow \mathrm{pK}^{+} \Sigma^{0} \rightarrow \mathrm{pK}^{+} \Lambda \gamma$ reaction. The average beam polarization of $(87.5 \pm 2.0) \%$ was determined for this measurement using the pp elastic scattering events analysis.

\section{Analysis and results}

The $\mathrm{pK}^{+} \Lambda$ events were analyzed to determine different physical observables in the reaction such as the Dalitz plot and angular distributions. The behavior of these observables is discussed in this paper.

The Dalitz plot is a useful tool to understand the reaction mechanism for reactions with three or more particles in the final state. The Dalitz plot in $m_{\mathrm{p} \Lambda}^{2}$ vs. $m_{\mathrm{K} \Lambda}^{2}$ is more suitable for the analysis of the $\overrightarrow{p p} \rightarrow \mathrm{pK}^{+} \Lambda$ reaction since different physical effects are predicted in $\{\mathrm{p} \Lambda\}$ and $\{\mathrm{K} \Lambda\}$ subsystems.

The Dalitz plot from measured $\mathrm{pK}^{+} \Lambda$ events at the excess energy $\varepsilon=204 \mathrm{MeV}$ is shown in Fig. 1 . The plot was corrected for the detector acceptance and reconstruction efficiency of about $20 \%$ determined by comparing the reconstructed and generated phase space distribution of the Monte Carlo simulation.

The resolution of the $\mathrm{p} \Lambda$ invariant mass was obtained to be $\sigma \approx 1.0 \mathrm{MeV} / c^{2}$. This high resolution of the $\mathrm{p} \Lambda$ invariant mass is mainly due to the performance of the high resolution straw tube tracker in the COSY-TOF detector which improved the mass resolution by a factor of $\approx 3$ compared to the previous setup of the COSY-TOF detector without the STT [5]. The Dalitz plot distribution shows that the COSYTOF detector covers the complete kinematically allowed region of the $\overrightarrow{p p} \rightarrow \mathrm{pK}^{+} \Lambda$ reaction. The Dalitz plot of the data deviates from homogeneity, so that significant enhancements are seen on the left side and in the center of the Dalitz plot. The strong enhancement at the center of the Dalitz plot is due to the so-called cusp effect which is seen in the $\{\mathrm{p} \Lambda\}$ subsystem. There are two $\mathrm{N} \Sigma$ cusp effects, a cusp at the $\mathrm{p} \Sigma^{0}$ threshold $\left(4.54 \mathrm{GeV}^{2} / c^{4}\right)$ and another cusp at the $\mathrm{n} \Sigma^{+}$threshold $\left(4.53 \mathrm{GeV}^{2} / c^{4}\right)$. The difference of these two cusp thresholds is about $1.98 \mathrm{MeV} / c^{2}$ which cannot be sep- 

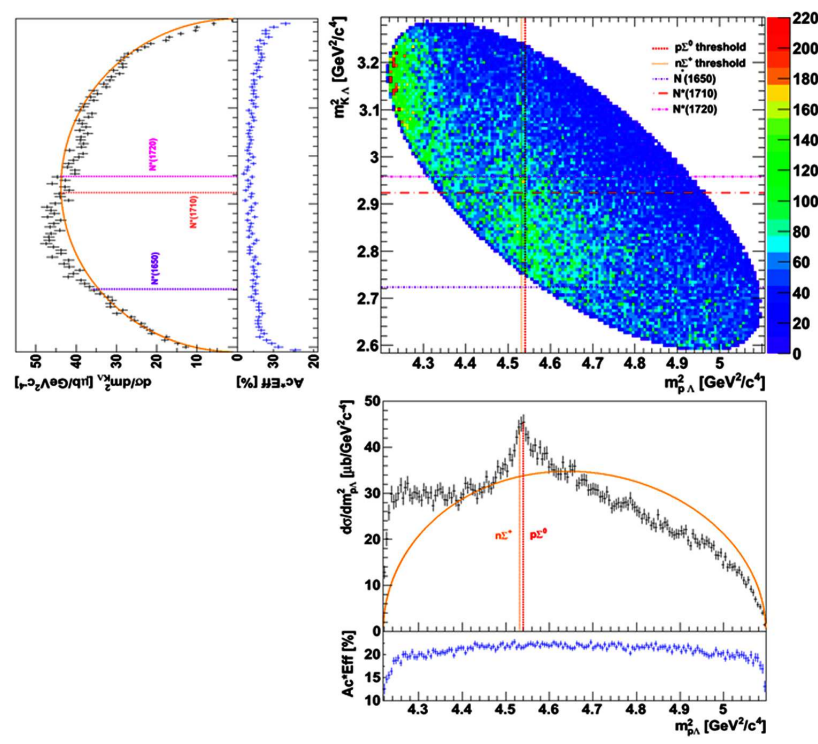

Fig. 1. Dalitz plot distribution and its projections for the measured $\mathrm{pK}^{+} \Lambda$ events at the excess energy $\varepsilon=$ $204 \mathrm{MeV}$ as a function of $m_{\mathrm{p} \Lambda}^{2}$ and $m_{\mathrm{K} \Lambda}^{2}$. The measured Dalitz plot clearly deviates from an isotropic distribution. The colored lines shows different effects involved in the reaction.

arated with the available statistics even with invariant mass resolution of about $1 \mathrm{MeV} / c^{2}$. Another strong enhancement is seen on the left side of the Dalitz plot due to the $\mathrm{p} \Lambda$ final state interaction at low $\mathrm{p} \Lambda$ invariant masses. Furthermore, the $\mathrm{N}^{*}(1650), \mathrm{N}^{*}(1710)$ and $\mathrm{N}^{*}(1720)$ resonances in the $\{\mathrm{K} \Lambda\}$ subsystem influence the Dalitz plot distribution. However, they are not seen as narrow structures in the Dalitz plot distribution due to their large width of about $100 \mathrm{MeV}$.

The angular distribution of the primary particles in the CMS is shown in Fig. 2 (left). All three distributions are corrected for the detector acceptance and reconstruction efficiency. The angular distribution of the particles in the CMS is symmetric around zero as predicted, since the particles in the entrance channel are identical. However, there is also some asymmetric behavior especially in the forward and backward direction which is due to the incomplete description of the detector at small angles in the Monte Carlo simulation. In the low energy region it is a good approximation to describe the angular distribution of the scattered particles by the Legendre polynomials $P_{l}(\cos \theta)$ up to second order which was fit to the distributions.

The angular distributions of the primary particles in the CMS are not directly related to the different contributions to the reaction mechanism. This can be seen in the helicity angular distributions of $\cos \theta_{\mathrm{Kp}}$ in the $\{\mathrm{p} \Lambda\}$ and $\{\mathrm{K} \Lambda\}$ rest frames and for $\cos \theta_{\Lambda \mathrm{p}}$ in the $\{\mathrm{pK}\}$ rest frame. The helicity distributions are shown in Fig. 2 (right) and corrected for the detector acceptance and reconstruction efficiency shown below the angular distributions. The physical effects in the reaction mechanism distort the isotropic behavior of the helicity angular distributions. The influence of resonances decaying into the $\{\mathrm{K} \Lambda\}$ subsystem introduces an enhancement at $\cos \theta_{\mathrm{Kp}}^{\mathrm{Rp} \Lambda}=-1$ in the $\{\mathrm{p} \Lambda\}$ rest frame and at the center of the $\{\mathrm{pK}\}$ rest frame. The $\mathrm{p} \Lambda$ final state interaction is also seen as a strong enhancement at $\cos \theta_{\mathrm{Kp}}^{\mathrm{RK} \Lambda}=-1$ in the
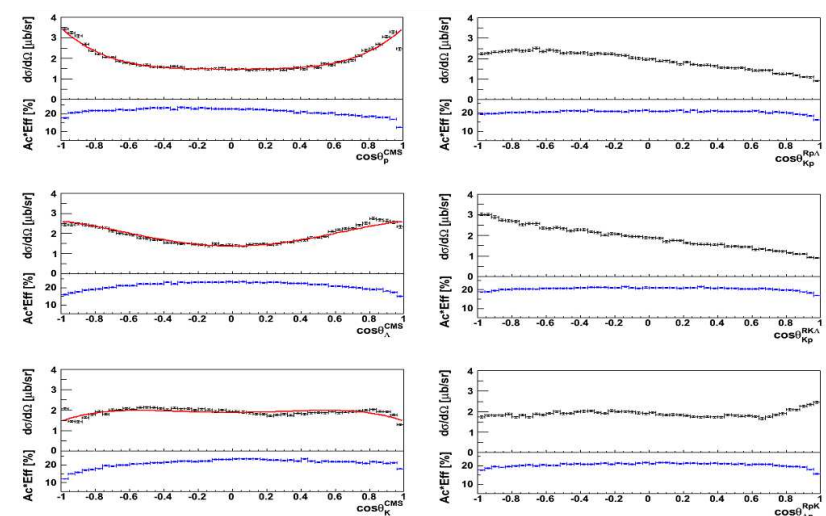

Fig. 2. Angular distributions of the primary particles in the CMS (left) and helicity frame (right) for the selected $\mathrm{pK}^{+} \Lambda$ events. The detector acceptance and reconstruction efficiency distribution is shown below each angular distribution. The red lines in left plot show

Legendre polynomial fits to the distributions.

$\{\mathrm{K} \Lambda\}$ rest frame and $\cos \theta_{\Lambda \mathrm{p}}^{\mathrm{RpK}}=1$ in the $\{\mathrm{pK}\}$ rest frame. The $\mathrm{N} \Sigma$ cusp effect did not introduce any enhancement in the helicity angular distribution. This is due to the dominant $S$-wave contribution in the angular distribution of the cusp effect $[2,6]$.

\section{Acknowledgments}

We acknowledge support by the Foundation for Polish Science-MPD program, co-financed by the European Union within the European Regional Development Fund and by the FFE grants of the Research Center Jülich.

\section{References}

[1] S. Abd El-Samad, M. Abdel-Bary, K. Brinkmann, H. Clement, J. Dietrich, E. Dorochkevitch, S. Dshemuchadse, K. Ehrhardt, A. Erhardt, W. Eyrich, C. Fanara, D. Filges, A. Filippi, H. Freiesleben, M. Fritsch, W. Gast, J. Georgi, A. Gillitzer, J. Gottwald, J. Hauffe, D. Hesselbarth, H. Jäger, B. Jakob, R. Jäkel, L. Karsch, K. Kilian, H. Koch, M. Krapp, J. Kreß, E. Kuhlmann, A. Lehmann, S. Marcello, S. Marwinski, Phys. Lett. B 688, 142 (2010).

[2] S. Jowzaee, Ph.D. Thesis, Jagiellonian University, Kraków 2014.

[3] F. Hauenstein, Ph.D. Thesis, University of ErlangenNürnberg, 2014.

[4] M. Roeder, E. Borodina, H. Clement, E. Doroshkevich, R. Dzhygadlo, K. Ehrhardt, A. Erhardt, W. Eyrich, W. Gast, A. Gillitzer, D. Grzonka, J. Haidenbauer, C. Hanhart, F. Hauenstein, P. Klaja, L. Kober, K. Kilian, M. Krapp, M. Mertens, J. Ritman, E. Roderburg, W. Schroeder, T. Sefzick, A. Sibirtsev, P. Wintz, P. Wüstner, Eur. Phys. J. A 49, 157 (2013).

[5] S. Abd El-Samad, E. Borodina, K.-Th. Brinkmann, H. Clement, E. Doroshkevich, R. Dzhygadlo, K. Ehrhardt, A. Erhardt, W. Eyrich, H. Freiesleben, W. Gast, A. Gillitzer, D. Grzonka, C. Hanhart, F. Hauenstein, P. Klaja, K. Kilian, M. Krapp, J. Ritman, E. Roderburg, M. Röder, M. SchulteWissermann, W. Schroeder, T. Sefzick, G.J. Wagner, P. Wintz, P. Wüstner, Eur. Phys. J. A 49, 41 (2013).

[6] S. Jowzaee et al., to be published, 2015. 